\title{
Bifurcation Phenomena in a Semiconductor Superlattice Subject to a Tilted Magnetic Field
}

\author{
Anton O. Selskii ${ }^{\mathrm{b}, \mathrm{a}}$, Alexander E. Hramov ${ }^{\mathrm{b}, \mathrm{a}}$, Alexey A. Koronovskii ${ }^{\mathrm{a}, \mathrm{b}, *}$, Olga \\ I. Moskalenko ${ }^{\mathrm{a}, \mathrm{b}}$, Alexander G. Balanov ${ }^{\mathrm{c}}$ \\ ${ }^{a}$ Saratov State University, Astrakhanskaya, 83, Saratov, 410012, Russia \\ ${ }^{b}$ Saratov State Technical University, Politehnicheskaja 77, Saratov 410056, Russia \\ ${ }^{c}$ Loughborough University, Loughborough LE11 3TU, United Kingdom
}

\begin{abstract}
The paper studies instabilities of charge transport in strongly coupled semiconductor superlattices with an applied electric and a tilted magnetic field. We reveal the bifurcation phenomena, which are associated with the transitions between different regimes of charge dynamics, and also investigate effects of the temperature on these bifurcations. In addition, we find out that the development of an instability can be accompanied by a graduate change of the dominant transport mechanism.
\end{abstract}

Keywords: pattern, semiconductor superlattice, bifurcation, tilted magnetic field, nonlinear dynamics

PACS: 05.45.-a, 73.21.-b, 05.45.Mt

\section{Introduction}

Semiconductor superlattices (SSLs), nanostructures formed by several alternating layers of different semiconductor materials [1, 2, 3, 4], are subjects of a great research interest for both the fundamental and applied sciences 1, 5, 6, 7,

5 8, [9, 10]. Being biased by an applied electric field they are able to demonstrate a large number of interesting quantum-mechanical phenomena such as WannierStark ladders, sequential and resonant tunneling, Bragg reflections, and Bloch

\footnotetext{
${ }^{*}$ Corresponding author

Email address: alexey.koronovskii@gmail.com (Alexey A. Koronovskii)
} 
oscillations. These phenomena strongly influence collective charge transport along the SSL inducing negative differential conductivity and traveling charge domains of high concentration [3]. In Ref. 11] it has been theoretically shown that two main types of charge domains can be generated in transferred electron devices with negative differential conductance, namely the pure accumulation domains and dipole domains. With this, Ref. 12] has reported on the detailed experimental study indicating that the current oscillations in superlattices are most likely occurring in the pure charge accumulation mode. Recently, it has been found out that a tilted magnetic field applied to a SSL can strongly affect the electron drift velocity in this nanostructure [13, 14] and, correspondingly, the dynamics of the SL in regime of charge domains propagation [15, 16].

From the viewpoint of collective charge dynamics, a SSL can be considered ${ }_{20}$ as an active nonlinear medium, where the spatio-temporal patterns (e.g. high concentration charge domains) can be generated by a voltage applied to the contacts of the SSL $[3,10]$. When the applied voltage is small, spatially extended patterns of charge concentration are stationary in time. For higher voltage the stationary state becomes unstable and charge domains start to propagate along the SSL, thus generating the current oscillations. One of the typical instabilities giving birth to the current oscillations in the SSL is the supercritical Hopf bifurcation [17, 18]. In this case, the current oscillations in the vicinity of the bifurcation are close to be harmonic. With further increase of the voltage, the shape and timescales both of the moving charge domains and the related current oscillations are considerably changed. In the absence of the magnetic field these modifications are rather gradual, whereas the presence of a tilted magnetic field seems to induce additional bifurcation phenomena, which are not clear at the moment [16].

In this paper we study the bifurcations induced by a tilted magnetic filed, 35 and investigate, how the change of temperature affects the instabilities. The structure of the paper is the following. Section 1 presents the mathematical model describing the charge transport in the SSL biased by an electric and a tilted magnetic field. The evolution of the charge dynamics with variation of the 
voltage applied is discussed in Sec. 2. The instabilities occurring in the system and the underlying mechanism for low temperatures are considered in Sec. 3 Section 4 is devoted to the transport regimes and the bifurcation phenomena at high temperatures. The final remarks are given in the Conclusions.

\section{Model equations}

In order to describe the collective charge dynamics in the SSL we use a set 45 of dimensionless current continuity and Poisson equations [19, 20]:

$$
\begin{gathered}
\frac{\partial n}{\partial t}=-\beta \frac{\partial J}{\partial x}, \\
\frac{\partial F}{\partial x}=\nu(n-1) .
\end{gathered}
$$

In Eqs. (10) and (2) the dimensionless volume electron density, electric field and current density are denoted as $n(x, t), F(x, t)$ and $J(x, t)$, respectively, $x$ and $t$ are the dimensionless space and time variables, $\beta=3.1 \times 10^{-2}, \nu=15.8$ are

${ }_{50}$ the dimensionless control parameters. The dimensionless quantities are related with the physical (primed) ones as:

$$
\begin{gathered}
x=x^{\prime} / L^{\prime}, \quad t=t^{\prime} / \tau^{\prime}, \quad n=n^{\prime} / n_{D}^{\prime}, \\
J=J^{\prime} /\left(e n_{D}^{\prime} v_{0}^{\prime}\right), \quad F=F^{\prime} / F_{c}^{\prime}, \quad F_{c}^{\prime}=\hbar /\left(e d^{\prime} \tau^{\prime}\right), \\
\beta=v_{0}^{\prime} \tau^{\prime} / L^{\prime}, \quad \nu=L^{\prime} e n_{D}^{\prime} /\left(F_{c}^{\prime} \varepsilon_{0} \varepsilon_{r}\right),
\end{gathered}
$$

where $d^{\prime}=8.3 \mathrm{~nm}$ and $L^{\prime}=115.2 \mathrm{~nm}$ are the period and the length of the superlattice, $e>0$ is the magnitude of the electron charge, $\Delta^{\prime}=19.1 \mathrm{meV}$ is the miniband width, $n_{D}^{\prime}=3 \times 10^{22} \mathrm{~m}^{-3}$ is the n-type doping density in the

55 SL layers, $F_{c}^{\prime}=3.2 \times 10^{5} \mathrm{~V} / \mathrm{m}$ is the normalization value of the electric field, $\varepsilon_{0}$ and $\varepsilon_{r}=12.5$ are the absolute and relative permittivities, respectively. The quantity:

$$
v_{0}^{\prime}=\gamma \frac{\Delta^{\prime} d^{\prime}}{2 \hbar} \frac{I_{1}(\Theta)}{I_{0}(\Theta)}
$$

is the maximal possible value of the dimensionless drift velocity without the tilted magnetic field, where

$$
\Theta=\Delta^{\prime} /\left(2 k_{B}^{\prime} T^{\prime}\right)
$$


characterizes the temperature $T^{\prime}$, while $I_{0}(x)$ and $I_{1}(x)$ are the modified Bessel functions of the first kind. Parameters $\gamma=\left[\tau_{e}^{\prime} /\left(\tau_{e}^{\prime}+\tau_{i}^{\prime}\right)\right]^{1 / 2}$ and $\tau^{\prime}=\gamma \tau_{i}^{\prime}$ are determined by the scattering events. These parameters depend on the elastic $\tau_{e}^{\prime}$ and inelastic $\tau_{i}^{\prime}$ scattering times. In our study we use the following values: $\tau^{\prime}=250$ fs and $\gamma=1 / 8.5$. The values of the physical quantities are taken from 65 recent experimental works [14, 21].

Within the drift-diffusion approximation the dimensionless current density can be written as:

$$
J=n v_{d}(F)-D(F) \frac{\partial n}{\partial x},
$$

where $v_{d}(F)$ is the dimensionless electron drift velocity $\left(v_{d}=v_{d}^{\prime} / v_{0}^{\prime}\right)$ and $D(F)$ is the diffusion coefficient [3]:

$$
\begin{aligned}
& D(F)=v_{d}(F) d \frac{\exp (-\kappa F)}{1-\exp (-\kappa F)}, \\
& \kappa=\frac{\hbar}{k_{B}^{\prime} T^{\prime} \tau^{\prime}}=\frac{\hbar \Theta}{\Delta^{\prime}}, \quad d=\frac{d^{\prime}}{L^{\prime}} .
\end{aligned}
$$

70 The diffusion coefficient (7) may be neglected when $T^{\prime} \rightarrow 0(\Theta \rightarrow \infty)$. If there is no tilted magnetic field, the drift velocity $v_{d}(F)$ is governed by the Esaki-Tsu formula [1], which in its dimensionless form can be written as:

$$
v_{d}(F)=\frac{F}{1+F^{2}} .
$$

In this case the dependence of the drift velocity on the electric field demonstrates only two extrema at $F_{c}= \pm 1$ (Esaki-Tsu peaks). In the presence of a tilted magnetic field the drift velocity $v_{d}(F)$ for an arbitrary temperature can be obtained numerically, e.g. using the approach described in [16]. In our calculations we apply a magnetic field $B^{\prime}=15 \mathrm{~T}$ tilted with respect to the SL axis $x$ at an angle of $\alpha=40^{\circ}$.

The calculated dependencies $v_{d}(F)$ for different $\Theta$ are shown in Figure 1(a). 80 One can see that for all temperatures the $v_{d}(F)$ curves exhibit multiple maxima. The first maximum observed for the lowest value of $F=F_{c}$ is the Esaki-Tsu peak, which is associated with the onset of the Bloch oscillations. Nonlinear interaction between the electronic Bloch oscillations along the SL and cyclotron 
motion in the plane of the layers induces chaotic semiclassical electron dynamics, which, depending on the ratio between the Bloch and cyclotron frequencies, either accelerate or decelerate charge transport through the SL [13, 14]. As a consequence we observe other maxima on the dependencies $v_{d}(F)$ corresponding to the Bloch-cyclotron resonances, which occur due to the resonant acceleration of the electrons whenever the ratio of the Bloch and cyclotron frequencies equals $r=0.5,1,2, \ldots$ (Bloch-cyclotron resonances) [13]. Thus, at the presence of a tilted magnetic field there are two major transport mechanisms, namely the conventional Esaki-Tsu transport [1] and the Bloch-cyclotron resonances, when the Bloch and cyclotron frequencies are commensurate, the electrons exhibit a unique type of quantum chaos, which does not obey the Kolmogorov95 Arnold-Moser theory [22]. This type of chaos is characterized by the formation of intricate web-like structures, known in the literature as "stochastic webs" [22, 23], which extend throughout the phase space of the miniband electrons. The appearance of these webs abruptly delocalizes electrons in real space, thus significantly increasing their drift velocity due to nonlinear interaction between the Bloch oscillations and cyclotron motion [13, 15].

Remarkably, as the temperature increases ( $\Theta$ decreases), the Esaki-Tsu peak dramatically weakens, whereas the resonant peaks become more prominent [16]. Moreover, new resonant peaks arise from the background with the drop of $\Theta$ (compare curves 2, 3 and 4 ).

105

The dimensionless bias (constant) voltage $V=V^{\prime} /\left(F_{c}^{\prime} L^{\prime}\right)$ applied to the SSL creates a global constraint:

$$
V=U+\int_{0}^{1} F d x,
$$

where the voltage $U$ across the contacts includes the effect of the charge accumulation and depletion at the emitter and collector and the contact resistance [24]. The integration in (9) is performed over the dimensionless length of the system under study, which is equal to 1 . In our study we consider a simple model of semiconductor superlattice without an external resonance circuit (Although 

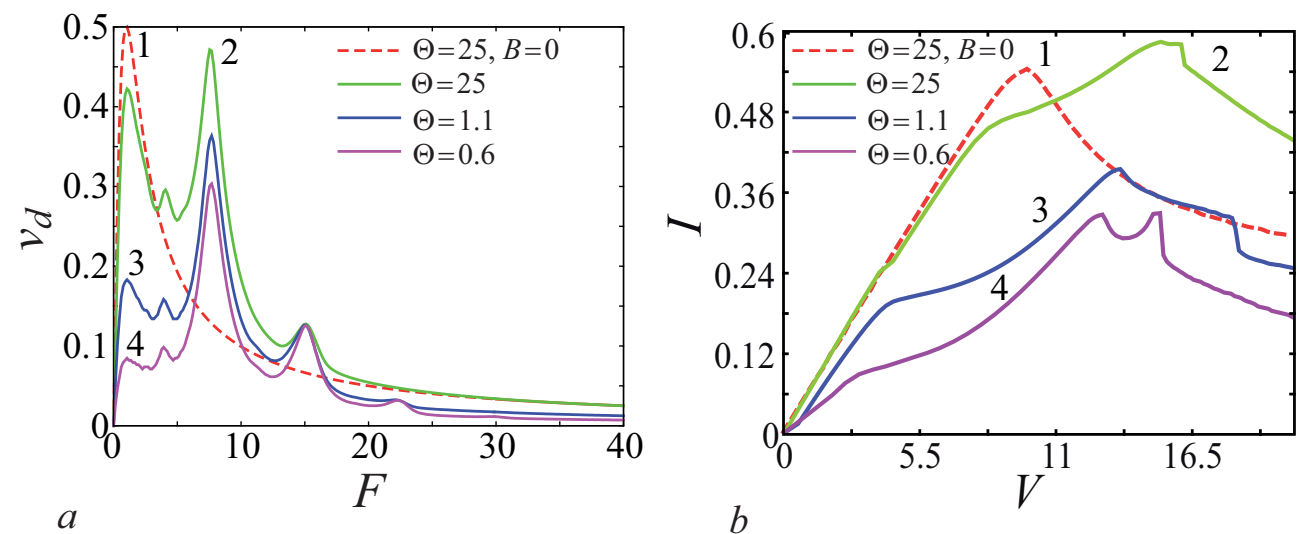

Figure 1: The dependences $v_{d}(F)$ (a) and $I(V)$-characteristics (b) for the case without the tilted magnetic field (the dashed line 1) and with a tilted magnetic field (solid lines 2-4). The curves 1 and 2 correspond to the parameter value $\Theta=\Theta_{1}=25\left(T^{\prime}=4.2 \mathrm{~K}\right)$, curve 3 to $\Theta=\Theta_{2}=1.1\left(T^{\prime}=100 \mathrm{~K}\right)$, and curve 4 to $\Theta=\Theta_{3}=0.6\left(T^{\prime}=200 \mathrm{~K}\right)$

the external voltage source connected to the superlattice may be considered as the simplest external circuit). Some questions of the influence of external resonance circuit on charge domains dynamics in superlattice has been considered in Refs. 12, 21, 10, 25].

To determine the dimensionless current density in the heavily doped emitter contact with electrical conductivity $\sigma^{\prime}=3788.0 \mathrm{Sm}^{-1}$ we use Ohmic boundary condition on the left boundary of the superlattice:

$$
J(0, t)=s F(0, t)
$$

where $s=\sigma^{\prime} F_{c}^{\prime} /\left(e n_{D}^{\prime} v_{0}^{\prime}\right)=17.7$ is the dimensionless control parameter corresponding to the electrical conductivity of the emitter contact. Such condition together with Eq. (91) is sufficient for the determination of current on the right boundary $J(1, t)$ of the system under study.

The model described by Eqs. (11) - (10) exhibits both constant and oscillating electric current, depending on the constant bias voltage, $V$, applied to SL. Fig. 1(b) shows calculated current-voltage $I(V)$-characteristics for the case without a tilted magnetic field and with a tilted magnetic field. For bias volt- 
ages $V$ at which current oscillations are observed, the DC current was calculated by averaging current oscillations $I(t)$ over time. When $B=0$ the $I(V)$-characteristic reveal the usual Esaki-Tsu-like behavior, characterized by a single maximum, which is associated with the onset of single-electron Bloch oscillations throughout much of the SL charge transport region. The electron dynamics changes dramatically when a tilted magnetic field is applied to the SL. All of the current-voltage characteristics reveal clear Bloch-cyclotron resonances, which manifest themselves through the appearance of additional features in the curves. For low temperatures, the Bloch-cyclotron resonances produce sudden changes in the slope of the $I(V)$-dependence and also shift the position of the current peak. As temperature increases, these effects become more prominent and, eventually, give rise to additional maxima in the $I(V)$ curves, e.g., the double peaks at $T=200 \mathrm{~K}$. This evolution originates from the variation of the $v_{d}(F)$-dependencies with changing temperature, shown in Fig. 1(a) (see also [16]).

\section{Charge dynamics in semiconductor superlattices in the absence of magnetic field}

The current oscillations in the SSL typically demonstrate a relaxation character. This manifests itself in the dynamics that is highly inhomogeneous in time. However, for values of $V$ slightly above the Hopf bifurcation (in the case of the absence of magnetic field $V_{c} \cong 10.1$ ), the current oscillations are almost harmonic. In order to study the transition from the nearly harmonic to relaxation oscillations with variation of $V$, we consider the evolution of the time series $I(t)$ and surface plots of $n(x, t)$. To better illustrate the changes in the current oscillations we also calculated the projections of the phase trajectories onto the plane $(I(t), I(t+\delta t))$ (where $\delta t$ is the time delay) obtained using the time-delay coordinates (Takens) approach [26]. The corresponding plots for the case without magnetic field $(B=0)$ and $\Theta=25\left(T^{\prime}=4.2 \mathrm{~K}\right)$ are presented in 

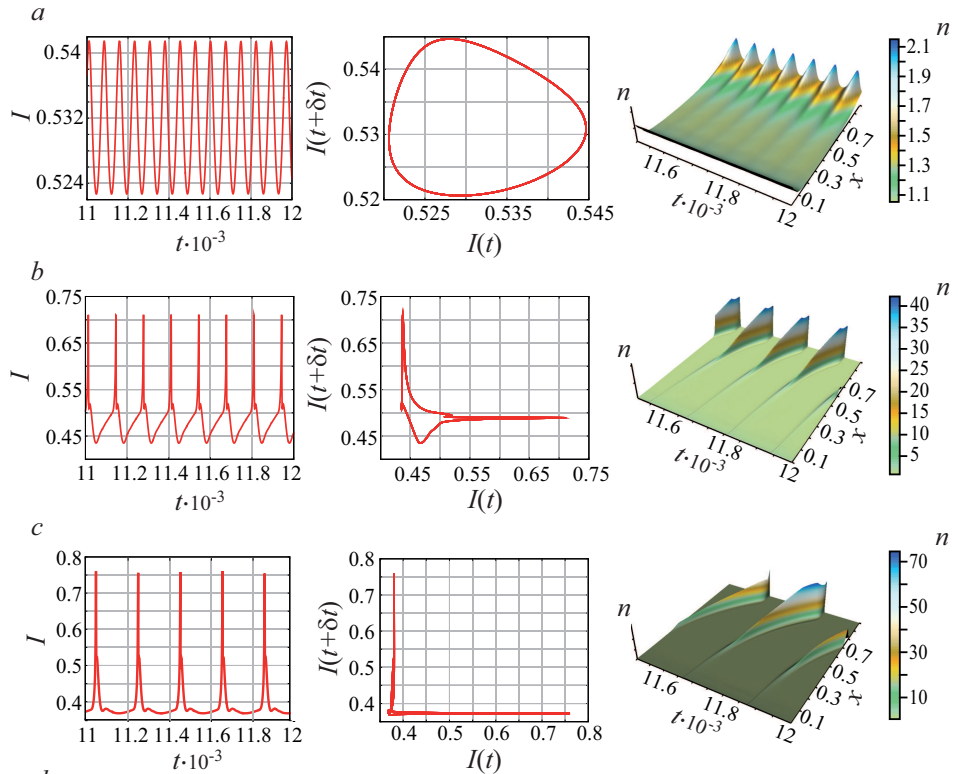

$d$
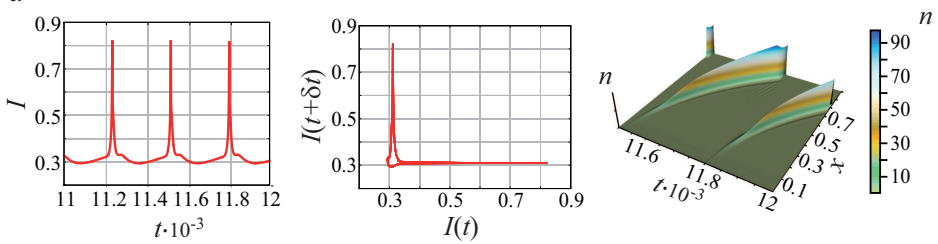

Figure 2: Dependence $I(t)$, projection of the phase trajectory onto the reconstructed phase space $(I(t), I(t+\delta t))$, spatio-temporal dynamics of $n(x, t)$ in the case without magnetic field for temperature $T=4.2 \mathrm{~K}\left(\Theta_{1}=25\right)$, and for $(a) V=10.1, \delta t=18 ;(b) V=11.2, \delta t=33.3$; (c) $V=13.5, \delta t=52 ;(d) V=16.8, \delta t=71.5$

$(I(t), I(t+\delta t))$ (central panels) reveals that although the amplitude, frequency and shape of the current oscillations change with variation of $V$, their topology remains the same, thus indicating no bifurcations. This is also confirmed by the spatio-temporal patterns of $n(t, x)$ (right panels), which for all $V$ demonstrate qualitatively the same character. We found similar behaviour for different values of $\Theta$, which allows us to conclude that in the absence of a magnetic field increase of $V$ does not induce any bifurcations. 


\section{Instabilities in semiconductor superlattices with an applied electric and a tilted magnetic field}

165 magnetic field is applied to the SSL. The presence of magnetic field results in the growth of the threshold value of generation. It becomes $V_{c}=15.5$ for fixed $\Theta=\Theta_{1}=25\left(T^{\prime}=4.2 \mathrm{~K}\right)$ when the SSL is biased by a magnetic field $B^{\prime}=15 \mathrm{~T}$ tilted with respect to the SSL axis $x$ at an angle of $\alpha=40^{\circ}$.

Figure 3 illustrates evolution of $I(t)$, the projection $(I(t), I(t+\delta t))$ and $n(t, x)$ with change of $V$ and fixed $\Theta, B^{\prime}$ and $\alpha$. For small $V=15.5$ the shape of the current oscillations is close to harmonic, and the projection of the phase trajectory onto the plane $(I(t), I(t+\delta t))$ demonstrates a smooth closed curve (see left and central panels in Figure $3(a))$. The dynamics of $n(t, x)$ shows moving domains of high charge density, which form highly ordered periodic patterns (see right panel of Figure [3 $(a)$ ). However, increase of $V$ up to $V_{c 1}=15.8$ changes the topology of both $I(t)$ and $n(t, x)$. As it is illustrated in Figure $3(b)$, although the shape of $I(t)$ (left panel) remains close to harmonic, the current oscillations start to demonstrate two peaks per period, and the period of the oscillations becomes twice as long. These changes are even more evident in

the shape of the phase trajectory (middle panel), which now demonstrates a double-loop limit cycle comparing with the single loop curve for the case of $V=15.5$. All these observations suggest that the system undergoes a period doubling bifurcation as voltage changes from $V=15.5$ to $V=15.8$. In terms of the spatio-temporal patterns of $n(t, x)$, this instability manifests itself in the branching of the propagating charge domain within the spatial interval $x \in$ $[0.3,0.7]$ (right panel in Figure $3(b)$ ). With further increase of $V$ the current oscillations become more inhomogeneous in time, but their topology remains the same, see Figure 3 $(c, d)$ and $(e)$, which illustrate the current oscillations and spatio-temporal patterns of $n(t, x)$ for $V=16.4(c), V=16.8(d)$ and $V=22.4(e)$, respectively.

In order to understand the relation between the development of the period- 

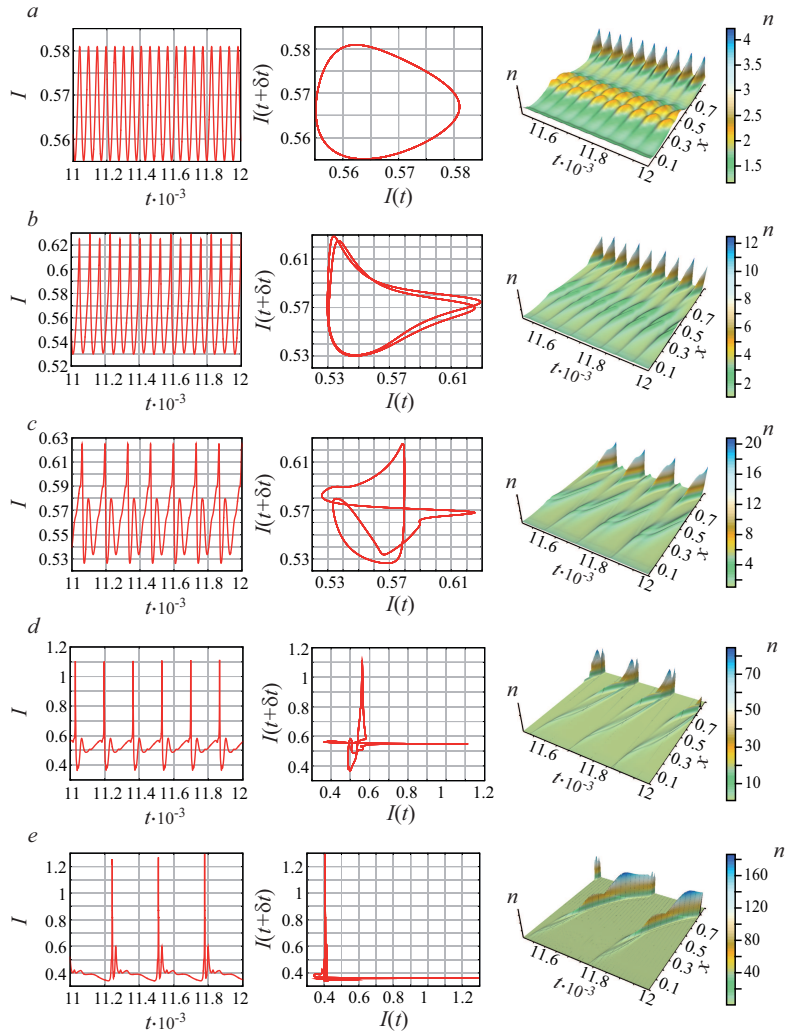

Figure 3: Dependence $I(t)$, projection of the phase trajectory onto the reconstructed phase space $(I(t), I(t+\delta t))$, spatio-temporal dynamics of $n(x, t)$ in the case with a tilted magnetic field for temperature $T^{\prime}=4.2 \mathrm{~K}\left(\Theta_{1}=25\right)$ and $(a) V=15.5, \delta t=13.1 ;(b) V=15.9, \delta t=$ $14.3 ;(c) V=16.4, \delta t=33.6 ;(d) V=16.8, \delta t=41.8 ;(e) V=22.4, \delta t=67.0$

doubling instability and the transport mechanisms realised in the SSL, we consider the probability distributions, $\rho(F)$, of the electric field, $F$, values. These distributions were calculated for all values of the electric field $F(x, t)$ along the superlattice space $(0 \leq x \leq 1)$ during one period, $T$, of current oscillations $\left(t_{1} \leq t<t_{1}+T, t_{1}\right.$ is the arbitrarily chosen time moment). Figure 4 shows the dependence of $\rho(F)$ together with the drift velocity-field characteristics, $v_{d}(F)$, for the case before $(a)$ and after the the period doubling bifurcation $(b)$. In both cases $\rho(F)$ demonstrates multiple peaks, which are in a good agreement with the peaks of $v_{d}(F)$. This reflects the fact that the propagating charge 


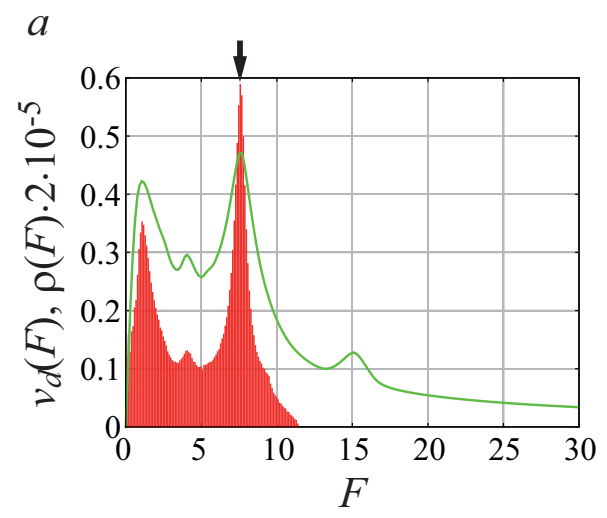

$b$

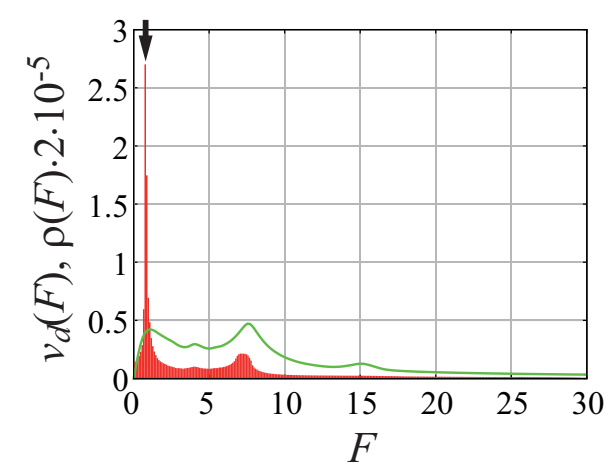

Figure 4: The profile $v_{d}(F)$ (solid line) and the distributions $\rho(F)$ (filled area plot) in the case of a tilted magnetic field for $\Theta=\Theta_{1}=25$ and $(a) V=15.5 ;(b) V=16.8$. Arrow indicates the dominant peak in $\rho(F)$.

domain is usually accumulated around $x$, where the local electric field $F(x, t)$ corresponds to one of the maxima in the $v_{d}(F)$-curve [19]. However, if before the bifurcation (for $V=15.5$ ) the position of the most prominent peak of $\rho(F)$ coincides with the position of the maximum of $v_{d}(F)$, which corresponds to the Bloch-cyclotron resonance with $r=1$, after the bifurcation (for $V=16.8$ ) the highest peak of $\rho(F)$ coincides with the peak of $v_{d}(F)$, which corresponds to the onset the Bloch oscillations (the Esaki-Tsu peak). This suggests that the development of the instability associated with the period-doubling bifurcation is accompanied by the change of the dominant transport mechanics, which is the Bloch-cyclotron resonance before the bifurcation, and the Esaki-Tsu transport after the bifurcation. Note, that in the case without magnetic field, when only Esaki-Tsu transport is realised in the system, we did not observe any bifurcations with increase of $V$ (see Section 2).

Figure 1 indicates that increase of temperature can significantly affect the structure and the heights of the peaks in $v_{d}(F)$ curve. It also influences on the threshold of Hopf bifurcation, i.e. it becomes slightly smaller in comparison with the case of low temperature considered above $\left(V_{c}=13.9\right.$ for $\Theta=\Theta_{2}=1.1$ $\left(T^{\prime}=100 \mathrm{~K}\right), V_{c}=13.2$ for $\left.\Theta=\Theta_{3}=0.6\left(T^{\prime}=200 \mathrm{~K}\right)\right)$. Therefore, to 

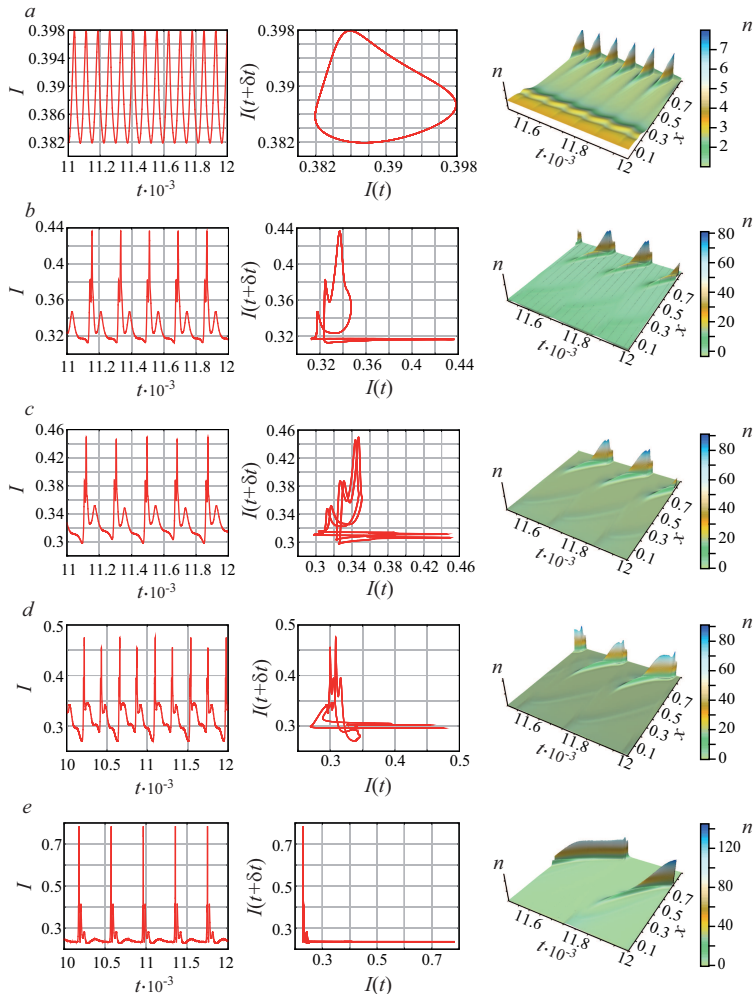

Figure 5: Dependence $I(t)$, projection of the phase trajectory onto the reconstructed phase space $(I(t), I(t+\delta t))$, spatio-temporal dynamics of $n(x, t)$ in the case with a tilted magnetic field for temperature $T^{\prime}=100 \mathrm{~K}\left(\Theta=\Theta_{2}=1.1\right)$ and $(a) V=13.9, \delta t=18.5$; $(b) V=$ $16.8, \delta t=45 ;(c) V=17.3, \delta t=48 ;(d) V=18.5, \delta t=109.7 ;(e) V=19.6, \delta t=97.5$

examine whether the change of dominant transport mechanisms associated with the period doubling instability is robust against the temperature variations, we analyse the current oscillations $I(t)$ and spatio-temporal patterns $n(t, x)$ for smaller $\Theta$. Figure 5 displays the evolution of $I(t)$, the projection of the phase trajectories onto the plane $(I(t), I(t+\delta t))$ and $n(t, x)$ for $\Theta=\Theta_{2}$ and different $V$. The plots indicate that the drop of $\Theta$ down to $\Theta_{2}$ does not destroy the period-doubling bifurcation. However, in contrast to the case $\Theta=\Theta_{1}$, the current oscillations, which are highly homogeneous near the threshold of generation (see Figure $5(a)$ ), first become inhomogeneous (Figure 5 (b)), and only then undergo the period doubling bifurcation (Figure 5 (c)). Moreover, 

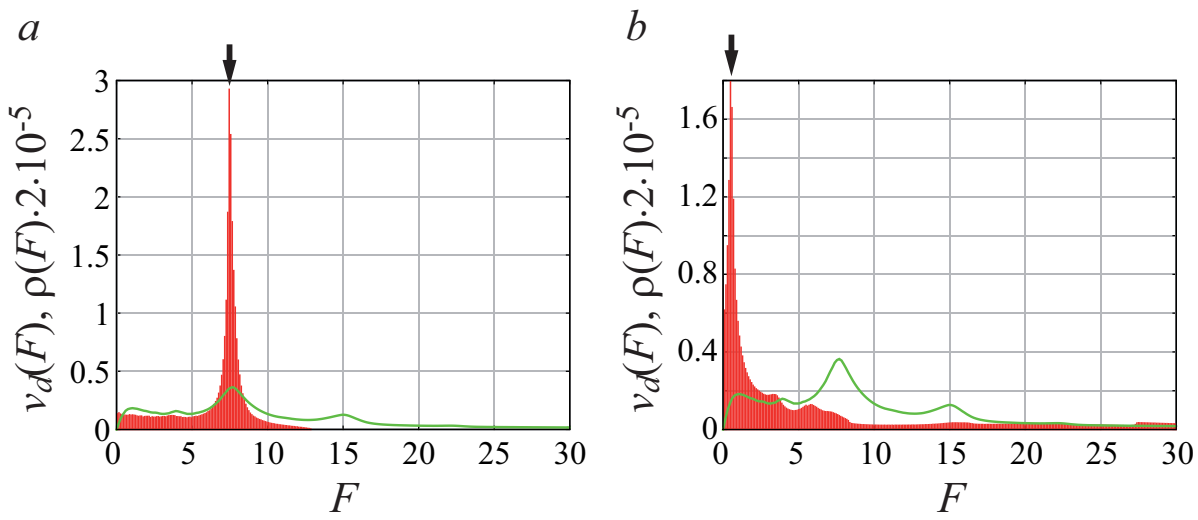

Figure 6: The profile $v_{d}(F)$ (solid line) and the distributions $\rho(F)$ (filled area plot) in the case of a tilted magnetic field for $\Theta=\Theta_{2}=1.1$ and for $(a)$ ( $a$ ) $V=13.9$; $(b) V=19.6$ Arrow indicates the dominant peak in $\rho(F)$.

the bifurcation takes place for $V$ larger than in the case $\Theta=\Theta_{1}$. As before, from the viewpoint of spatio-temporal dynamics of $n(t, x)$, the period doubling bifurcation is reflected in the branching of the propagating charge domains. Further increase of $V$ makes the oscillations more inhomogeneous, but does not produce any other instabilities (Figure $5(d)$ and $(e)$ ).

We calculated the distribution $\rho(F)$ for $V=13.9$ before the bifurcation and for $V=16.8$ after the bifurcation, and its shape with the profile of $v_{d}(F)$ for $\Theta=\Theta_{2}$ (see Fig. 6). Although the height of the Esaki-Tsu peak for $\Theta=\Theta_{2}$ is smaller than in the case of $\Theta=\Theta_{1}$ (compare curves 2 and 3 in Fig. 1), it is still quite prominent and plays an important role in the generation of the collective charge transport. This is confirmed by Fig. [6 which shows that, as in the case of lower temperature $\Theta_{1}$, before the bifurcation the most prominent peak of $\rho(F)$ takes place at the $F$ value, corresponding to the Bloch-cyclotron resonance with $r=1$. However, after the bifurcation the most probable value of $F$ correspond to the Esaki-Tsu peak. This concludes that such a change of dominant transport mechanism associated with period-doubling bifurcation is a robust phenomenon which can be realised within a range of temperatures. 


\section{4. "Death" of the current oscillations}

As Figure 1 shows, the increase of the temperature can eventually lead to the situation when the Esaki-Tsu peak in $v_{d}(F)$ becomes indistinct (curve 4 ). instability we calculated $I(t)$ and $n(t, x)$ for $\Theta=\Theta_{3}$ and different values of $V$. The corresponding plots are presented in Figure 7 As in the previous cases discussed above, the oscillations born via the Hopf bifurcation are homogeneous near the threshold of generation (Figur $7(a))$. However, the increase of $V$ up 255 to $V=V_{c 1}^{\Theta_{3}}=15.2$ leads to oscillation "death" [16] caused by an inverse Hopf bifurcation. Thus, in the range of $V \in\left[V_{c 1}^{\Theta_{3}}, V_{c 2}^{\Theta_{3}}\right]$, where $V_{c 2}^{\Theta_{3}}=15.7$, the SSL does not demonstrate current oscillations (Figure7 $(b)$ ). In this case the spatial distribution of the charge concentration is stationary, i.e does not depend on time: $n(t, x) \equiv n(x)$. Despite of its stationary character, $n$ is highly Figur $7(b)$ ). Further increase of $V$ restores the oscillations (Figure7 $(c)$ ), which appear again due to the Hopf instability developed. Remarkably, for this value of $\Theta=\Theta_{3}$ the period doubling instability is not developed for the physically meaningful range of voltages $V \in[0,22.2]\left(V^{\prime} \in[0,0.8] \mathrm{V}\right)$.

265 trated in Figure 8, Similar to the cases considered in Section 3, for small $V$ the $\rho(F)$ curve demonstrates a pronounced maximum at the value of $F$ corresponding to the Bloch-cyclotron resonance (Figure $8(a)$ ). With onset of the oscillation death (Fig. $8(b))$ the shape of $\rho(F)$-distribution changes only slightly, developof oscillations with increase of $V$ significantly deform $\rho(F)$, which demonstrates multiple peaks with the larger one not associated with any of peaks in the corresponding curve $v_{d}(F)$. 

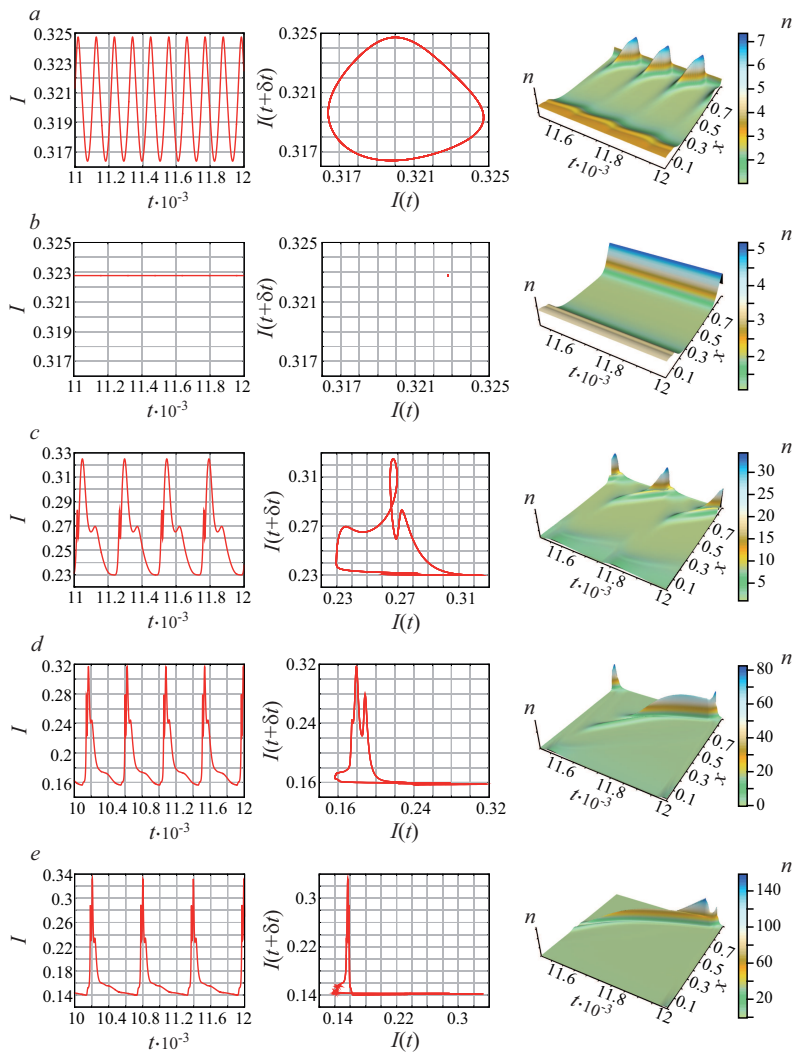

Figure 7: Dependence $I(t)$, projection of the phase trajectory onto the reconstructed phase space $(I(t), I(t+\delta t))$, spatio-temporal dynamics of $n(x, t)$ in the case with a tilted magnetic field for temperature $T^{\prime}=200 \mathrm{~K}\left(\Theta=\Theta_{3}=0.6\right)$ and $(a) V=13.2, \delta t=26.8 ;(b) V=15.4$; (c) $V=15.7, \delta t=62 ;(d) V=19.6, \delta t=114.1 ;(e) V=22.2, \delta t=148.5$

\section{Conclusion}

In conclusion, we have revealed the electric instabilities, which can be developed in strongly coupled SSLs subjected to a tilted magnetic field. The onset and the extinction of the current oscillations induced by charge domains moving along the SSL are typically associated with the Hopf bifurcation both for the case of absence [17] and presence of the magnetic field [19]. However, a tilted magnetic field can induce an additional period doubling instability, whose development with variation of $V$ is accompanied by change of the dominant transport 
a

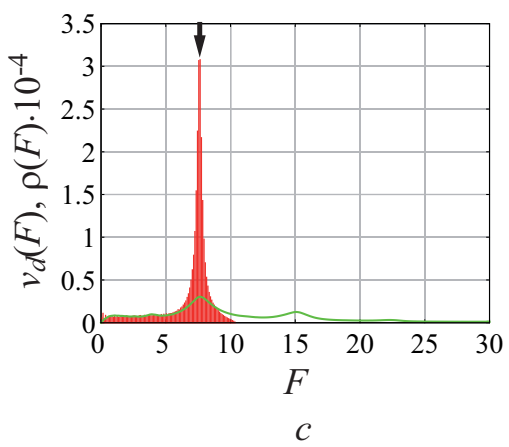

b

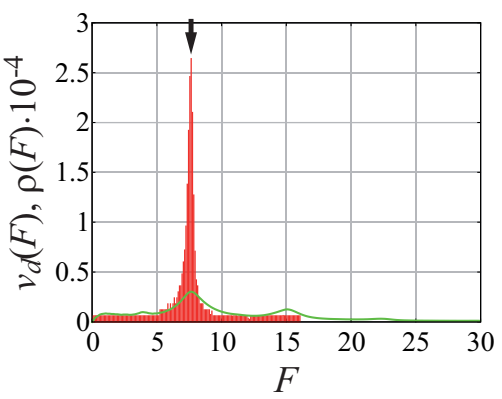

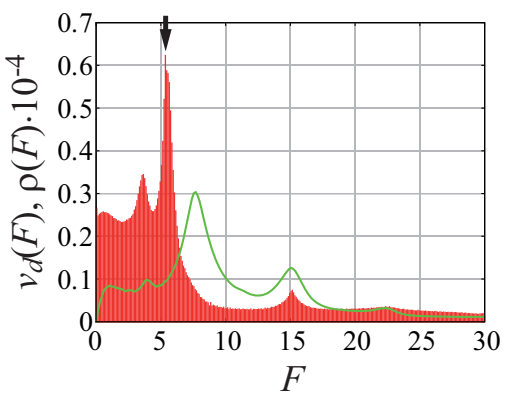

Figure 8: The profile $v_{d}(F)$ (solid line) and the distributions $\rho(F)$ (filled area plot) obtained in the presence of a tilted magnetic field for $\Theta=\Theta_{3}=0.6$ and for $(a) V=13.2 ;(b) V=15.7$; (c) $V=19.6$. The $v_{d}$-curve contains several peaks depending on the applied voltage $V:(a)$ the only one well-pronounced peak corresponding to the resonance 1:1 between Bloch and cyclotron frequencies; $(b)$ two peaks corresponding to 1:1 and 2:1 resonances; $(c)$ four peaks corresponding to Esaki-Tsu peak, 1:2, 1:1 and 2:1 resonances. Arrow indicates the dominant peak in $\rho(F)$-distribution

mechanism. Before this bifurcation (for lower $V$ ) the resonant Bloch-cyclotron dynamics [13, 19] plays a leading role in the charge transport along the SSL. However, with the development of the period doubling instability (for higher $V$ ) the conventional Esaki-Tsu transport [1] starts to dominate. This instability exists within a certain temperature range, but for high enough temperature the period doubling bifurcation disappears. Our results suggest that the bifurcation phenomena can be utilised for controlling the dominant transport mechanisms in SSLs. Since these mechanisms crucially affect the amplitude and frequency 
19, 21], the results presented are useful for development and design of sub- $\mathrm{THz}$ sources that use the SSLs as key elements.

\section{Acknowledgments}

We thank referees of the paper for valuable suggestions and comments. Russian Science Foundation supported study of the semiconductor superlattice coupled to resonator (Grant No. 14-12-00222). A.A.K. acknowledges financial support from the Ministry of Education and Science of Russian Federation (Grant No. $3.59 .2014 / \mathrm{K})$.

\section{References}

[1] L. Esaki, R. Tsu, Superlattices and negative differential conductivity in semiconductors., IBM Journal of Research and Development 14 (1) (1970) $61-65$.

[2] A. Y. Shik, Superlattices-periodic semiconductor structures, Sov. Phys. Semicond. 8 (1975) 1195.

[3] A. Wacker, Semiconductor superlattices: a model system for nonlinear transport, Physics Reports 357 (2002) 1-111.

[4] R. Tsu, Superlattices to Nanoelectronics, Elsevier, 2005.

[5] Y. Romanov, Nonlinear effects in periodic semiconductor structures, Optika i Spektroskopiya 33 (1972) 917.

[6] A. A. Ignatov, V. I. Shashkin, Bloch oscillations of electrons and instability of space-charge waves in superconductor superlattices, Sov. Phys. JETP 66 (3) (1987) 526.

[7] M. Holthaus, Collapse of minibands in far-infrared irradiated superlattices, Physical Review Letters 69 (1992) 351. 
[8] Y. Zhang, J. Kastrup, R. Klann, K. H. Ploog, H. T. Grahn, Synchronization and chaos induced by resonant tunneling in GaAs/AlAs superlattices, Physical Review Letters 77 (1996) 3001.

[9] T. Hyart, J. Alexeeva N. V. Mattas, K. N. Alekseev, Terahertz bloch oscillator with a modulated bias, Phys. Rev. Lett. 102 (2009) 140405.

320 [10] A. E. Hramov, A. A. Koronovskii, S. A. Kurkin, V. V. Makarov, M. B. Gaifullin, K. N. Alekseev, N. Alexeeva, M. T. Greenaway, T. M. Fromhold, A. Patane, F. V. Kusmartsev, V. A. Maximenko, O. I. Moskalenko, A. G. Balanov, Subterahertz chaos generation by coupling a superlattice to a linear resonator, Phys.Rev.Lett. 112 (2014) 116603.

[11] H. Kroemer, Negative conductance in semiconductors, IEEE Spectrum 5 (1) (1968) 47-56. doi:10.1109/MSPEC.1968.5215632.

[12] K. F. Renk, B. I. Stahl, Operation of a semiconductor superlattice oscillator, Physics Letters A 375 (27) (2011) 2644-2651. doi:http://dx.doi.org/10.1016/j.physleta.2011.05.038.

[13] T. M. Fromhold, A. A. Krokhin, C. R. Tench, S. Bujkiewicz, P. B. Wilkinson, F. W. Sheard, L. Eaves, Effects of stochastic webs on chaotic electron transport in semiconductor superlattices, Phys. Rev. Lett. 87 (4) (2001) 046803. doi:10.1103/PhysRevLett.87.046803.

[14] T. M. Fromhold, A. Patane, S. Bujkiewicz, P. B. Wilkinson, D. Fowler, D. Sherwood, S. P. Stapleton, A. A. Krokhin, L. Eaves, M. Henini, N. S. Sankeshwar, F. W. Sheard, Chaotic electron diffusion through stochastic webs enhances current flow in superlattices, Nature 428 (2004) 726-730.

[15] A. G. Balanov, D. Fowler, A. Patane, L. Eaves, T. M. Fromhold, Bifurcations and chaos in semiconductor superlattices with a tilted magnetic field, Phys. Rev. E 77 (2) (2008) 026209.

[16] A. O. Selskii, A. A. Koronovskii, A. E. Hramov, O. I. Moskalenko, K. N. Alekseev, M. T. Greenaway, F. Wang, T. M. Fromhold, A. V. Shorokhov, 
N. N. Khvastunov, A. G. Balanov, Effect of temperature on resonant electron transport through stochastic conduction channels in superlattices, Phys. Rev. B 84 (2011) 235311.

[17] J. Hizanidis, A. G. Balanov, A. Amann, E. Schöll, Noise-induced front motion: Signature of a global bifurcation, Phys. Rev. Lett. 96 (24) (2006) 244104. doi:10.1103/PhysRevLett.96.244104.

[18] K. N. Alekseev, A. G. Balanov, A. A. Koronovskii, V. A. Maximenko, O. I. Moskalenko, A. E. Hramov, Stability of the steady state in a strongly coupled semiconductor superlattice described using a semiclassical approach, Bulletin of the Russian Academy of Sciences: Physics 77 (12) (2013) 14441447.

[19] M. T. Greenaway, A. G. Balanov, E. Schöll, T. M. Fromhold, Controlling and enhancing terahertz collective electron dynamics in superlattices by chaos-assisted miniband transport, Phys. Rev. B 80 (2009) 205318.

[20] A. A. Koronovskii, A. E. Hramov, V. A. Maximenko, O. I. Moskalenko, K. N. Alekseev, M. T. Greenaway, T. M. Fromhold, A. G. Balanov, Lyapunov stability of charge transport in miniband semiconductor superlattices, Phys. Rev. B 88 (2013) 165304.

[21] N. Alexeeva, M. T. Greenaway, A. G. Balanov, O. Makarovsky, A. Patane, M. B. Gaifullin, F. V. Kusmartsev, T. M. Fromhold, Controlling highfrequency collective electron dynamics via single-particle complexity, Phys. Rev. Lett. 109 (2) (2012) 024102.

[22] R. Z. Sagdeev, D. A. Usikov, G. M. Zaslavsky, Nonlinear Physics, Harwood Academic Publishers, NY, 1988.

[23] G. M. Zaslavsky, R. Z. Sagdeev, D. A. Usikov, A. A. Chernikov, Weak Chaos and Quasi-Regular Patterns, Cambridge University Press, Cambridge, 1991. 
[24] M. T. Greenaway, A. G. Balanov, T. M. Fromhold, Controlling Charge Domain Dynamics in Superlattices, Wiley-VCH Verlag GmbH \& Co. KGaA, 2012.

[25] V. V. Makarov, A. E. Hramov, A. A. Koronovskii, K. N. Alekseev, V. A. Maksimenko, M. T. Greenaway, T. M. Fromhold, O. I. Moskalenko, A. G. Balanov, Sub-terahertz amplification in a semiconductor superlattice with moving charge domains, Applied physics letters 106 (2015) 043503-1043503-4.

[26] F. Takens, Detecting strange attractors in dynamical systems and turbulence, in: D. Rand, L.-S. Young (Eds.), Lectures Notes in Mathematics, N. Y.: Springler-Verlag, 1981, p. 366. 